\title{
Correction to: Existence of eigenvalues embedded in the spectral bands of Schrödinger operators on carbon nanotubes with impurities
}

\author{
Hiroaki Niikuni ${ }^{1}$ \\ Published online: 11 October 2019 \\ ๑) Springer Nature B.V. 2019
}

\section{Correction to: Letters in Mathematical Physics https://doi.org/10.1007/s11005-019-01220-9}

The original version of the article unfortunately contained a mistake. In the second line in Abstract, "Parchment" should be "Kuchment and Post". The author overlooked this mistake, which occured in an editorial process after the manuscript has been accepted. The author should read the proof more carefully. He would like to apologize to them sincerely.

Publisher's Note Springer Nature remains neutral with regard to jurisdictional claims in published maps and institutional affiliations.

The original article can be found online at https://doi.org/10.1007/s11005-019-01220-9.

Hiroaki Niikuni

niikuni@maebashi-it.ac.jp

1 Maebashi Institute of Technology, 460-1 Kamisadori, Maebashi City, Gunma 371-0816, Japan 